Luciano BARDI, Hanspeter KRIESI and Alexander H. TRECHSEL (eds), 2014, Elections in Europe in Times of Crisis. Contributions from the 2013 EUDO Dissemination Conference, Florence, European University Institute, eBook.

Jessica Sainty

\title{
OpenEdition
}

Journals

Édition électronique

URL : http://journals.openedition.org/ress/2982

DOI : $10.4000 /$ ress. 2982

ISSN : $1663-4446$

Éditeur

Librairie Droz

Édition imprimée

Date de publication : 15 mai 2015

Pagination : 303-305

ISSN : 0048-8046

Référence électronique

Jessica Sainty, « Luciano BARDI, Hanspeter KRIESI and Alexander H. TRECHSEL (eds), 2014, Elections in Europe in Times of Crisis. Contributions from the 2013 EUDO Dissemination Conference, Florence,

European University Institute, eBook. », Revue européenne des sciences sociales [En ligne], 53-1 | 2015, mis en ligne le 30 mars 2015, consulté le 22 septembre 2020. URL : http://journals.openedition.org/ ress/2982 ; DOI : https://doi.org/10.4000/ress.2982

Ce document a été généré automatiquement le 22 septembre 2020

(c) Librairie Droz 


\section{Luciano BARDI, Hanspeter KRIESI and Alexander H. TRECHSEL (eds), 2014, Elections in Europe in Times of Crisis. Contributions from the 2013 EUDO Dissemination Conference, Florence, European University Institute, eBook.}

Jessica Sainty

1 Cet ouvrage collectif, issu de deux journées d'études organisées à Florence, en novembre 2013, par l'European Union Democracy Observatory, dresse un état des lieux de la démocratie en Europe à partir de l'analyse des élections qui s'y déroulent dans le prolongement de la crise économique de 2008. Un état des lieux qui, à la veille du scrutin européen de 2014, semble particulièrement nécessaire aux éditeurs de l'ouvrage. Ces élections sont en effet à la fois les premières après l'entrée en vigueur du traité de Lisbonne, les premières à donner lieu à l'élection du président de la Commission européenne par les députés - dont les auteurs espèrent qu'elle conférera plus de légitimité aux politiques impulsées par la Commission -, et les premières à avoir lieu après une crise économique - dont ils soupçonnent qu'elle favorise l'émergence de formes nouvelles d'euroscepticisme.

Les dix contributions de l'ouvrage, regroupées en cinq sections, sont articulées autour de questionnements variés offrant un « panorama » de réponses à la très large question initiale : quels sont les facteurs influençant les résultats électoraux, quel est l'impact de la crise économique au niveau individuel dans les élections nationales et dans celles européennes, quel est son impact sur les systèmes partisans nationaux, quel est le 
degré d'européanisation des élections nationales ou de nationalisation des élections européennes?

3 La première section est consacrée au cas italien, analysant le rôle de l'identification partisane dans la construction des préférences politiques des électeurs (chapitre 1) et les reconfigurations organisationnelles des partis politiques italiens, impulsées par le processus amorcé de dénationalisation de la vie politique italienne, au travers des procédures de sélection des candidats présentés aux différentes élections (chapitre 2).

4 La deuxième section de l'ouvrage se focalise sur les effets de la crise économique en testant l'hypothèse d'un "vote économique" des électeurs des pays des Balkans (chapitre 3), puis celle d'un recours accru des électeurs néerlandais aux formes de participation non conventionnelles (chapitre 4).

5 La troisième section se concentre sur les élections européennes de 2014 (à venir au moment de la rédaction de l'ouvrage). Un chapitre propose une étude des récentes reconfigurations institutionnelles de l'UE (chapitre 5) ; le suivant s'interroge sur la congruence entre les perceptions des problèmes économiques par les électeurs européens et par la classe politique, ces différences de points de vue pouvant être l'une des explications possibles du déficit démocratique de l'UE (chapitre 6).

6 La quatrième section questionne les effets de la crise économique sur les systèmes partisans européens. Une première contribution montre comment l'intégration européenne, malgré la crise économique, exerce des pressions fortes dans le sens de la démocratisation. Dans les anciens pays appartenant au bloc soviétique (chapitre 7) l'argument européen est mobilisé en tant que tel par les partis du camp démocrate, en opposition au camp autoritaire. Une deuxième contribution revisite les travaux de Peter Mair sur la faible influence de l'intégration européenne sur les systèmes partisans nationaux. Elle montre comment l'importance de plus en plus grande de l'UE dans le policy making au niveau national est l'une des raisons de l'expression vigoureuse d'un euroscepticisme sur la scène politique, de plus en plus audible de la part des électeurs (chapitre 8). Une troisième contribution explore le lien entre l'attachement à la protection sociale et le vote d'extrême-droite, en montrant que le sentiment d'insécurité économique et les valeurs de justice et de solidarité sociales peuvent paradoxalement se renforcer et conduire à voter en faveur de l'extrême-droite, dans une logique de défense du système de protection sociale (chapitre 9).

7 L'unique contribution de la cinquième et dernière section, sous la plume de Simona Piattoni, interroge l'impact des élections sur les perceptions de la démocratie représentative dans un système "multi-niveaux " ? Ces élections à différents niveaux ont en effet engendré une confusion chez les électeurs quant aux attributions respectives du niveau européen et du niveau national. Cette confusion serait, pour l'auteure, l'une des causes du déficit démocratique, puisque les citoyens n'évalueraient pas leurs différents représentants en fonction de leur réelle activité politique (chapitre 10).

8 La lecture de l'ouvrage, assez homogène, séduit par la richesse des analyses présentées et des cas étudiés, ainsi que par l'actualisation qu'il propose de l'étude des élections en Europe. Son point fort réside dans la variété des terrains observés. L'Europe y est entendue au sens large, c'est-à-dire à la fois l'UE mais également certains des pays candidats à l'entrée dans l'UE ou des pays voisins (les pays de l'ex-Yougoslavie, la Moldavie ou la Géorgie, par exemple). Leur étude apparaît particulièrement importante pour comprendre les voies de leur stabilisation démocratique et pour commencer à 
constituer des bases de données électorales. Ces pays restent relativement peu étudiés en comparaison des anciennes démocraties occidentales, alors qu'ils risquent d'avoir une influence considérable sur les dynamiques futures de l'intégration européenne.

9 Néanmoins, comme beaucoup d'ouvrages collectifs issus de colloques ou de conférences, celui-ci a les défauts de ses qualités. La grande diversité des contributions qui y sont rassemblées nuit à la cohérence générale de l'ensemble. Si les élections en Europe sous l'influence de la crise économique sont le sujet principal, le questionnement spécifique à chaque contribution est très variable, passant des élections nationales aux européennes, des pays membres de l'UE à des pays nonmembres, des approches par l'identification partisane au vote économique, etc.

Cette réserve faite, plusieurs contributions présentent des pistes de réflexions stimulantes et tout particulièrement celle du chapitre $1:$ « Do Parties still Orient Voters in Times of Crisis? Evidence of Partisan Cueing Effects in 2013 Italy ", sous la plume de Lorenzo De Sio, Aldo Paparo, Joshua A. Tucker et Ted Brader. Elle applique les apports de la psychologie sociale et des travaux fondateurs d'Angus Campbell sur l'identification partisane à l'entrée sur la scène politique de nouveaux partis critiques de la classe politique et de l'austérité comme le Movimento 5 Stelle (M5S) de l'humoriste Beppe Grillo. À partir d'une approche expérimentale qui teste l'attribution des propositions programmatiques aux différents partis politiques, les auteurs entendent démontrer que les partis continuent de jouer un rôle de repère dans la construction des préférences politiques des électeurs italiens, y compris des partis nouvellement arrivés comme le M5S. Si cette contribution permet d'actualiser la théorie de l'identification partisane, si elle réhabilite le rôle des partis politiques face aux reproches récurrents qui leur sont adressés en termes de cartellisation ou de déconnexion trop grande vis-àvis des préoccupations des électeurs, elle suscite néanmoins quelques interrogations. On peut reprocher aux auteurs une vision un peu idéalisée de la démocratie, selon laquelle les électeurs disposeraient d'une connaissance solide des programmes des partis politiques et fonderaient leurs raisonnements électoraux sur une comparaison raisonnée des différentes offres politiques en compétition à un moment donné. C'est passer vite sur les inégalités de compétence politique des électeurs et la pluralité des mécanismes cognitifs conduisant à l'élaboration d'un choix électoral. Si le caractère expérimental de l'enquête menée par les auteurs du chapitre 1 est très stimulant, le cadrage normatif de l'analyse est révélateur d'un problème plus large des études rassemblées dans l'ouvrage. Tout en soulevant des pistes de réflexion extrêmement riches, elles sont parfois trop contraintes théoriquement pour déchiffrer de manière satisfaisante la complexité des défis auxquels est actuellement confrontée la démocratie en Europe.

11 Enfin, les contributions rassemblées dans cet ouvrage proposent des analyses quantitatives qui gagneraient à être prolongées et approfondies par une approche qualitative. Celle-ci permettrait de mieux appréhender le phénomène de l'euroscepticisme, par exemple, ou de dépasser la notion de vote protestataire par un examen plus fouillé du ressentiment que peuvent nourrir certains électeurs à l'égard de l'intégration européenne, particulièrement dans le contexte de la crise économique aigue de 2008 dont les effets perdurent. 


\section{AUTEURS}

\section{JESSICA SAINTY}

Sciences Po Grenoble - PACTE 